\title{
Clinical Utility of the Behavioral Pain Assessment Tool in Patients Admitted in the Intensive Care Unit
}

\author{
Sukanya Mitra ${ }^{1}$, Kompal Jain ${ }^{2}$, Jasveer Singh ${ }^{3}$, Puja Saxena ${ }^{4}$, Tenzin Nyima ${ }^{5}$, Selwin R Selvam ${ }^{6}$, Mansi C Walia ${ }^{7}$
}

\begin{abstract}
Introduction: Unnoticed and unrelieved pain is one of the main sources of psychological and physiological stress for intensive care unit (ICU) patients. The eight-item behavior pain assessment tool (BPAT) is a multicountry validated tool to assess pain in ICU patients. However, its feasibility and clinical utility for ICU patients in India need further research.

Aims and objectives: The Aims and objectives of the study were to assess pain using BPAT and its clinical utility in pain assessment and management in ICU patients.

Materials and methods: Following ethical approval, 400 consecutive adult patients admitted in the ICUs in a tertiary care teaching hospital were assessed for pain severity using BPAT at intake, baseline pain and procedural pain. Patients $<18$ years and in deep coma on the Glasgow coma scale were excluded from the study. The patients with BPAT score $\geq 4$ were given opioid analgesic, and their pain was reassessed after 2-3 hours. A feedback regarding feasibility and clinical utility was filled by the doctors.

Results: High interrater agreement for BPAT was observed with excellent kappa coefficients $(>0.85)$ for each item. The BPAT significantly guided the pain management $(p<0.0001)$. More than $90 \%$ of doctors found BPAT easy to understand and use. In most of the cases $(95.5 \%)$, doctors agreed that BPAT can improve the clinical management of ICU patients.

Conclusion: The BPAT is a reliable, brief, and an easy-to-use pain assessment tool, which clinicians can use for guiding pain assessment and management in the ICU setting on a routine basis.

Clinical significance: We recommend implementing BPAT in the clinical practice for better pain assessment and control in ICU patients.

Keywords: Assessment, Intensive care unit, Management, Pain, Scale, Utility.

Indian Journal of Critical Care Medicine (2020): 10.5005/jp-journals-10071-23521
\end{abstract}

\section{INTRODUCTION}

Unrelieved pain has long been identified as one of the greatest concerns for patients admitted in the intensive care unit (ICU). Majority of patients (80\%) experience different intensities of pain during their ICU stay. ${ }^{1}$

The pain during ICU stay could be attributed to constant background pain due to surgical and medical causes and intermittent or periprocedural pain. ${ }^{2}$ The main ICU procedures causing pain as evaluated in Europain study are turning, positioning, respiratory exercises, mobilization, intravenous and arterial line insertion, endotracheal (ET) suction, chest tube removal, and wound drain removal. Although the adverse effects of procedural pain (PP) have not received sufficient research attention, it has been demonstrated that PP, as a type of acute pain, can be a threat to tissue integrity, initiating a series of psychological, physiologic, and inflammatory stress responses. ${ }^{3}$

Owing to sedation, altered consciousness, and endotracheal intubation, many patients are unable to effectively communicate their pain through self-report. Unnoticed and unrelieved pain is one of the main sources of psychological and physiological stress for ICU patients. It can further result in harmful multisystem consequences and can therefore impair a patient's recovery and discharge. Therefore, it is essential that clinicians should have valid and reliable pain assessment methods for ICU patients.

The eight-item Behavior Pain Assessment Tool (BPAT) is one of the latest scales to assess pain in critically ill patients with high reliability $(r=0.54, p<0.001)$, discriminant, criterion, and convergent validity established across 28 countries, and sensitivity

\begin{abstract}
${ }^{1-7}$ Department of Anaesthesia and Intensive Care, Government Medical College and Hospital, Chandigarh, India

Corresponding Author: Sukanya Mitra, Department of Anaesthesia and Intensive Care, Government Medical College and Hospital, Chandigarh, India, Phone: +91172-2665253, e-mail: drsmitra12@ yahoo.com

How to cite this article: Mitra S, Jain K, Singh J, Saxena P, Nyima T, Selvam SR, et al. Clinical Utility of the Behavioral Pain Assessment Tool in Patients Admitted in the Intensive Care Unit. Indian J Crit Care Med 2020;24(8):695-700.

Source of support: ISCCM. REF. ISCCM/2017-18/42, dated July 20, 2017 Conflict of interest: None
\end{abstract}

and specificity ranging from 61.8 to $75.1 \% .{ }^{4}$ However, the feasibility and clinical utility of BPAT in assessing pain in different ICU patient groups including in India need further research. The clinical utility and feasibility are the important indicators for implementation of the scale in daily practice. ${ }^{5} \mathrm{~A}$ scale or instrument may be valid and psychometrically sound, and may be excellent for use for research purposes, but it may be complex, lengthy, time-consuming, difficult to interpret or score, intensive training-dependent, or with such other characteristics that make it unsuitable for clinical application on a routine basis. Further, in order to make a clinical tool useful for day-to-day use in a busy and resource-intensive setting like ICU, it must be perceived to be useful for guiding clinical management of patients and for directing outcome. These are the characteristics that are clubbed under the constructs "feasibility" and "clinical 
utility," which are considered to be essential characteristics of a scale before it can have practical clinical application. ${ }^{6}$ Many pain scales have undergone such feasibility and clinical utility studies. ${ }^{5,7,8}$ In fact, the authors of the BPAT study themselves highlighted the need to further study the "feasibility for use in practice and the effect of its clinical implementation on patient pain and intensive care unit outcomes." ${ }^{\prime 4}$ This is particularly relevant for developing countries. ${ }^{9}$ This was the rationale for this study.

The aim of the study was to assess pain using BPAT and its clinical utility in pain assessment and management in ICU patients. The primary objective of the study was to assess clinical utility of BPAT in assessing baseline pain (BP) and PP and its management in ICU patients. The secondary objectives of the study were to assess clinical utility and feasibility of BPAT using a feedback questionnaire, studying interrater reliability, and perceived usefulness of BPAT in guiding postprocedural pain management.

\section{Materials and Methods}

A prospective survey with repeated measures design was conducted between June 2019 and February 2020 in a tertiary teaching hospital in Chandigarh. After taking approval from the institutional ethical committee (ECR/658llnst/PB12014/RR-2017, dated May 15, 2018) and registration with Clinical Trials Registry — India [CTRI/2019/06/019691], 400 consecutive adult patients admitted in the ICUs were recruited in the study. The study design was an exploratory survey; hence, the inclusion criteria were kept broad by design to assess all the patients in the ICU irrespective of their diagnosis, treatment, or ability to verbalize. The patients with age less than 18 years and deep coma on the Glasgow Coma Scale (GCS) (score $\leq 3$ or less for nonintubated and nonventilated patients, and 1 each on eye movement and motor responses criteria for patients on ventilator or tracheal intubation) $)^{10}$ were excluded from the study. The study was ethically conducted in accordance with Declaration of Helsinki.

After obtaining a written informed consent either from the patient or kin, the patients were enrolled for the purpose of the survey. Demographic data of all the patients were noted. On admission to the ICU, sedation using either infusion morphine at the rate of $0.01-0.02 \mu \mathrm{g} / \mathrm{kg} / \mathrm{hour}$ or infusion fentanyl at the rate of $0.5-1 \mu \mathrm{g} / \mathrm{kg} / \mathrm{hour}$ and infusion midazolam at the rate of $0.03-0.05$ $\mathrm{mg} / \mathrm{kg}$ was started as per the institutional ICU protocol. Then, the patients were assessed for the pain severity using BPAT at intake (within 2-3 hours of admission). The pain assessment at intake (within 2-3 hours of admission) using BPAT was done separately by two senior resident doctors (one after the other, at the same time) on all the patients to study interrater reliability of BPAT. No training was given to them about BPAT prior to enrolment. The doctors were asked to note the ratings separately and not share with others.

The BPAT was repeatedly administered every day at a fixed time of the day (around 9 AM) to measure the BP of the patients. As per the suggested clinical guidelines by the authors of BPAT, ${ }^{4}$ the patients with BPAT score 4 or more were given appropriate opioid analgesic bolus unless clinically contraindicated. The pain of the patients was reassessed at 30-minute intervals up to 2-3 hours after the opioid administration using BPAT, to see lasting effect of the opioids guided by BPAT scoring.

Procedural pain was assessed using BPAT immediately prior and after the noninvasive and invasive painful ICU procedures in the patients. The procedures included turning, positioning, mobilization, intravenous and arterial line insertion, ET suction, chest tube removal, wound drain removal, respiratory exercises, and others. ${ }^{3}$ If the patients had BPAT score 4 or more, they were given appropriate opioid analgesic bolus and were reassessed after 15 minutes.

The structured observations regarding all conditions that might cause pain (original clinical diagnosis, complications, any addiction or withdrawal, and all procedures likely to cause pain) were recorded. The duration of ICU stay, pain intensity at time of discharge from $I C U,{ }^{11}$ and clinical outcome including morbidity and mortality were also recorded. A feedback of 10 questions was obtained from the doctors (four resident doctors) working in the ICU regarding their perceived clinical utility and feasibility of the BPAT in pain assessment and management of the patients in the clinical practice. The feasibility questionnaire included "ease of understanding the BPAT items"(1), "ease of applying BPAT on the patients,"(2) and "time spent on rating on BPAT."(3) The first two questions were rated on a five-point scale-"very easy, easy, medium, difficult, and very difficult." The time spent was rated on a three-point scale- "short, medium, and lengthy". The utility of applying BPAT (4) was rated on a five-point scale in various situations including patients with tracheostomy, on ventilator, paralyzed, delirious patients, and PP. The five-point scale included excellent, good, neutral, poor, and useless. Similarly, the clinical application of BPAT in directing analgesic therapy (5) was also rated on a similar five-point scale. The questionnaire also included specific feature of BPAT, which was most liked (6) and disliked (7). "Can BPAT improve the clinical management of ICU patients" (8) and "should BPAT be used routinely in the ICU for day-to-day patient assessment and management in the ICU" (9) were answered on a two-point scale-_yes" or "no." The last question was regarding any other comments about BPAT. (10)

The BPAT is a recent and multicountry validated instrument for assessing pain in the ICU. It was developed by Gélinas et al. in 2017. The scale was tested in 192 ICUs from 28 countries in 5 continents, with detailed psychometric properties and research validation. It has eight items assessing behavioral aspects of pain. It includes four facial expressions, two bodily responses, and two verbal responses. Facial responses include neutral expression, grimace, wince, and closed eyes accompanied by a picture. Bodily responses include muscle rigidity and clenched fists. Muscle rigidity is checked in both the upper and lower extremities. Moaning and verbal pain complaints are included in verbal responses. Each item has a dichotomous "present-absent" response. The "present" response of each item is marked as 1 and the total score ranges from 0 to 8 . It has been suggested that a score of $>3.5$ (i.e., 4 or more) indicates severe pain that should be treated with opioid analgesics. ${ }^{4}$ Pain intensity at the time of discharge was noted on numerical rating scale ranging from 0 to $10 .{ }^{11}$

Based on a conservative estimate and our own clinical experience of working in the ICU, at least $50 \%$ of the patients admitted in the ICU experience pain severe enough to clinically warrant opioid analgesic administration during some point of their stay in the ICU. With a precision of $5 \%$ and confidence interval of $95 \%$, the required sample size was 385 (calculated using the software sampsize) (available at http://sampsize.sourceforge.net/ iface/index.html\#prev). Thus, the sample size was set at 400 as a slightly overinclusive round figure.

The primary outcome of the study was to assess clinical utility of BPAT in assessing BP and PP. The secondary outcomes included feasibility and clinical utility of BPAT using feedback questionnaire, interrater reliability of BPAT, and duration of ICU stay.

The findings on the outcome measures were subjected to suitable parametric and nonparametric statistical analysis using IBM Statistical Package for the Social Sciences (SPSS) Statistics for 
Windows, Version 25 (IBM Corp., Armonk, NY, USA). ${ }^{12}$ Interrater reliability was studied by using BPAT for pain assessment by two senior resident doctors separately (one after the other, at the same time) on all patients, and the agreement between the two sets of scores using kappa coefficient was calculated. Nonparametric equivalent of repeated-measure ANOVA (Friedman's ANOVA) before and after opioid administration as dictated by BPAT for BP and PP was used to ascertain the clinical utility of the BPAT to guide pain management. $p<0.05$ was considered statistically significant.

\section{Results}

The mean age of the patients was $43.5 \pm 17.59$ years. Majority of the patients $(233 / 400 ; 58.25 \%)$ were male. Patients with various morbidities admitted in ICU were enrolled in the study (Fig. 1).

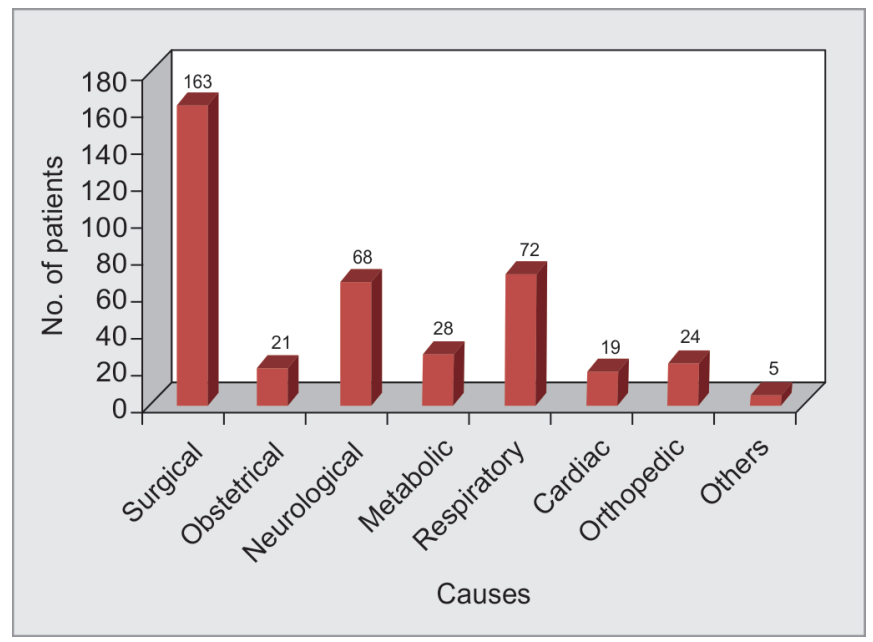

Fig. 1: Specialty-related causes for admission in ICU

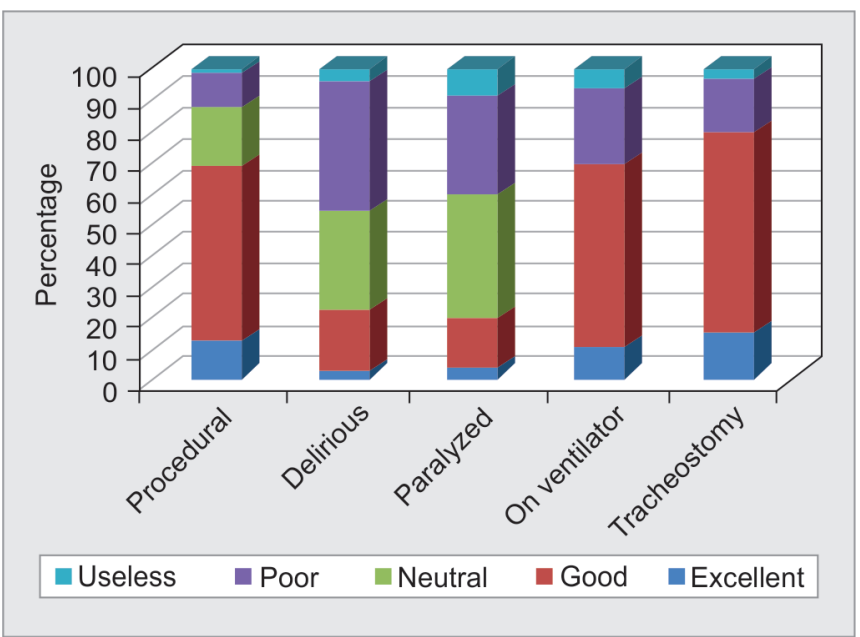

Fig. 3: Utility of BPAT in different situations

Table 1: Feedback regarding feasibility of applying BPAT in ICU
The median pain score at intake was 2 (IQR 2). Median BP score and median BP score after opioid treatment were 2 (IQR 2) and 2 (IQR1), respectively ( $p$ value $<0.0001$ ). Median preprocedural, procedural, and after treatment pain scores were 2 (IQR 1), 3 (IQR 2 ), and 2 (IQR 0$)$, respectively ( $p$ value $<0.0001$ ) (Fig. 2 ).

In our study, we prospectively evaluated the clinical utility of BPAT in assessment and management of both BP and PP in different subgroups of ICU patients in our current accepted institutional protocol ( $p$ value $<0.0001$ ) (Figs 3 and 4). Neutral expression $(50.6 \%)$ and closed eyes (46.8\%) followed by grimace (37\%) and wince (34.7\%) were the most frequent behaviors observed at intake and while assessing BP. Majority of clinicians found BPAT as a quick tool (59.25\%), which was easy to understand (93.3\%) and apply without any training (91.8\%) prior to enrolment (Table 1). The BPAT had

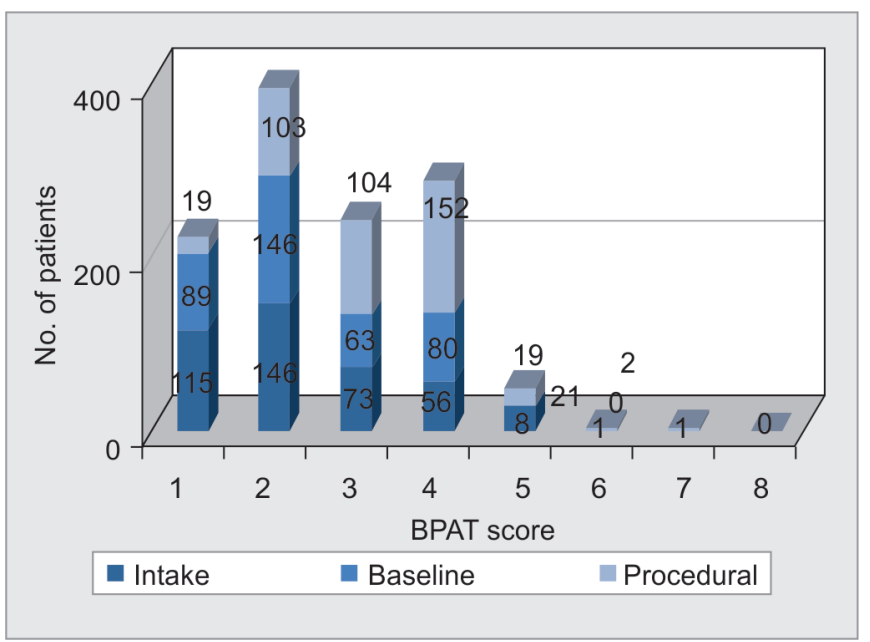

Fig. 2: Pain severity using BPAT at intake (2-3 hours after admission), BP (at $9 \mathrm{AM}$ ), and PP during ICU procedures

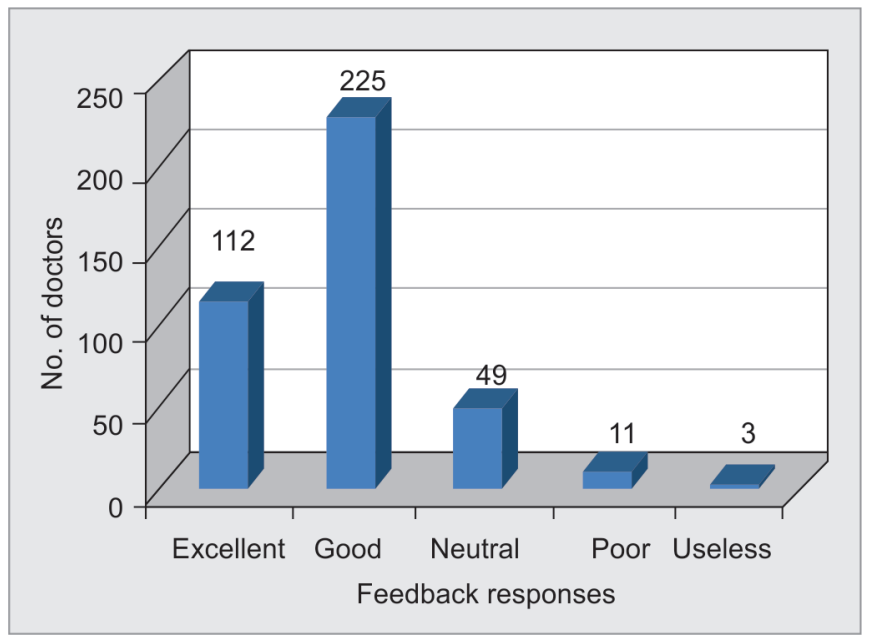

Fig. 4: Clinical application of BPAT in directing analgesic therapy

\begin{tabular}{|c|c|c|c|c|c|}
\hline \multirow[b]{2}{*}{ Feasibility } & \multicolumn{5}{|c|}{ Feedback responses } \\
\hline & Very easy & Easy & Medium & Difficult & Very difficult \\
\hline $\begin{array}{l}\text { Understanding the } \\
\text { BPAT items }\end{array}$ & $33 \%(132 / 400)$ & $60.3 \%(241 / 400)$ & $5.8 \%(23 / 400)$ & $0.5 \%(2 / 400)$ & $0.5 \%(2 / 400)$ \\
\hline $\begin{array}{l}\text { Applying BPAT on } \\
\text { patients }\end{array}$ & $36.3 \%(145 / 400)$ & $55.5 \%(222 / 400)$ & $7.8 \%(31 / 400)$ & $0.5 \%(2 / 400)$ & $0 \%(0 / 400)$ \\
\hline $\begin{array}{l}\text { Time spent on rating } \\
\text { on BPAT }\end{array}$ & $\begin{array}{l}\text { Short } 59.25 \% \\
(237 / 400)\end{array}$ & $\begin{array}{l}\text { Medium } 38.5 \% \\
(154 / 400)\end{array}$ & Long $2.25 \%(9 / 400)$ & - & - \\
\hline
\end{tabular}


highest perceived utility (marked as "excellent" or "good" responses) in tracheostomized patients, those on ventilator, and for PP, whereas utility was felt to be limited for paralyzed and delirious patients (Fig. 3). It also had a high proportion of good-to-excellent clinical application in directing the analgesic therapy (pharmacological interventions) (Fig. 4).

High interrater agreement for BPAT was observed for both the full scale and for each item individually, with excellent kappa coefficients ( 0.90 and $>0.85$, respectively) (Table 2 ).
Figures 3 and 4 and Tables 1 and 2 illustrate the feedback responses of the doctors regarding feasibility and clinical utility of BPAT. In most of the cases (feedback on 382 out of 400 patients; $95.5 \%$ ), doctors agreed that BPAT can increase the clinical management of ICU patients. Similarly, in most of the cases (372/400; 93\%), doctors felt that BPAT should be routinely used in the ICU for day-to-day patient assessment and management in the ICU.

Mean duration of ICU was $6.92 \pm 6.91$ days. The condition improved in $68.3 \%$ of the patients (273/400). Out of 400 patients,

Table 2: BPAT with interrater reliability for each item (kappa coefficient) (95\% confidence interval)

\begin{tabular}{|c|c|c|c|c|c|}
\hline Behavior & Definition & Frequency & $\begin{array}{l}\text { Interrater reliability } \\
\text { (kappa coefficient) } \\
\text { (95\% confidence } \\
\text { limit) }\end{array}$ & Liked response & $\begin{array}{l}\text { Disliked } \\
\text { response }\end{array}$ \\
\hline $\begin{array}{l}\text { Neutral expres- } \\
\text { sion }\end{array}$ & Muscles relaxed & $50.6 \%$ & $0.99(0.977-1.00)$ & $18.3 \% 73 / 400$ & $19.8 \% 79 / 400$ \\
\hline Grimace & $\begin{array}{l}\text { A sharp contortion } \\
\text { of the face } \\
\text { Au 4: Brow lowering } \\
\text { Au 7: Lid tightening } \\
\text { Au 6: Cheek raising } \\
\text { Au 20: Mouth } \\
\text { stretching } \\
\text { Au 43: Eye closing }\end{array}$ & $37 \%$ & $0.995(0.985-1.00)$ & $67.0 \% 268 / 400$ & $4.8 \% 19 / 400$ \\
\hline Wince & $\begin{array}{l}\text { To shrink away from } \\
\text { the start } \\
\text { Au 7: Lid tightening } \\
\text { Au 6: Cheek raising }\end{array}$ & $34.7 \%$ & $\begin{array}{l}0.961(0.932- \\
0.990)\end{array}$ & $2.0 \% 8 / 400$ & $1.5 \% 6 / 400$ \\
\hline Eyes closed & Lids are shut & $46.8 \%$ & $\begin{array}{l}0.890(0.855- \\
0.941)\end{array}$ & $4.0 \% 16 / 400$ & $59 \% 236 / 400$ \\
\hline Moaning & $\begin{array}{l}\text { Low soft indistin- } \\
\text { guishable sounds }\end{array}$ & $12.7 \%$ & 1.00 & $4.3 \% 17 / 400$ & $2.5 \% 10 / 400$ \\
\hline $\begin{array}{l}\text { Verbal com- } \\
\text { plaints }\end{array}$ & $\begin{array}{l}\text { Words used to } \\
\text { describe pain }\end{array}$ & $13.2 \%$ & 1.00 & $2.3 \% 9 / 400$ & $7 \% 28 / 400$ \\
\hline Muscle rigidity & $\begin{array}{l}\text { Stiff tensed muscles } \\
\text { of extremities }\end{array}$ & $16.2 \%$ & $\begin{array}{l}0.943(0.893- \\
0.988)\end{array}$ & $2.3 \% 9 / 400$ & $4.8 \% 19 / 400$ \\
\hline Clenched fists & Act of forming a fist & $11.6 \%$ & $\begin{array}{l}0.951(0.904- \\
0.998)\end{array}$ & $000 / 400$ & $0.8 \% 3 / 400$ \\
\hline
\end{tabular}

$\mathrm{Au}$, action unit; P/A, present/absent

Behavioral pain assessment tool (BPAT) (Copyright 2017: International Association for the Study of Pain) taken from Gelinas et al. ${ }^{4}$ 
23.3\% (93/400) died. The median NRS score of the patients was 2 (IQR 1). Majority of the patients $(287 / 307 ; 93.4 \%)$ had NRS score less than 2 on discharge from ICU.

\section{Discussion}

In this prospective study on 400 consecutive adult patients admitted in the ICUs in a tertiary care teaching hospital who were assessed for pain severity using BPAT at intake, BP and PP, high interrater agreement for BPAT was observed. The BPAT was perceived to be useful in guiding pain management. More than $90 \%$ of doctors found BPAT easy to understand and use. In most of the cases, doctors agreed that BPAT can improve the clinical management of ICU patients.

The most commonly used methods to assess pain in ICU patients are using objective measures like behavioral scales, despite new trends like pupillometry, skin conductance, and BIS index. ${ }^{13}$ It has been seen that the PP can be successfully measured using behavioral indicators in an Indian ICU. ${ }^{14}$ Studies have shown that behaviors such as grimace and wince were related to pain. ${ }^{4,15,16}$ The behavioral pain scale (BPS) and critical care pain observation tool (CPOT) are the commonly used tools for assessing pain in ICU. 13,14,17-

${ }^{21}$ However, the strength of existing research on nonpharmacologic approaches for PP is limited. ${ }^{22}$

Our results are in congruence with that of Gelinas et al. ${ }^{4}$ They too observed neutral expression most commonly at rest. They found high concordance, i.e., 89 and $82 \%$ before and during the procedure, respectively, between the two clinicians with good interrater reliability (0.43-0.60) for each item of BPAT. The least kappa coefficient was observed for behavior "muscle rigidity" as it was tested visually. ${ }^{4}$ However, in our study, the muscle rigidity was tested by moving the muscles of both upper and lower extremities. Rigidity observed in any extremity was marked as present.

The BPS and CPOT, unlike BPAT, consist of a confounding factor, i.e., compliance with mechanical ventilation, and complex training is required prior to use. ${ }^{13,18}$ In BPS, muscle rigidity is assessed visually whereas in CPOT it is tested while moving patients' arm. ${ }^{4,16,23,24}$ The CPOT and BPS also need further evaluation in delirious patients, patients with traumatic brain injury and painful ICU procedures (except positioning and tracheal suctioning), respectively. ${ }^{13,17,18,25}$ CPOT- Neuro has been derived from original CPOT for assessing pain in critically ill brain-injured patients. ${ }^{8}$ In some studies, validity and reliability of BPS were variable. ${ }^{13,18}$

The limitations of the BPAT were "closed eyes" behavior and its poor utility in ventilated paralyzed patients. The behavior "closed eyes" and "closing eyes" might have been interchanged resulting in lower kappa coefficient. They could also be associated with both analgesia and pain. The verbal and bodily responses could not be assessed in ventilated, paralyzed patients. Hence, BPAT could further be modified to facilitate the pain assessment in paralyzed intubated patients.

In our study, we assessed and managed BP using BPAT as preprocedural pain assessment and management could act as a preemptive analgesic intervention. ${ }^{3}$ In contrast, another study demonstrated that preprocedural pain assessments were performed only in $20 \%$ of 44 participating sites. Around 25 and $31 \%$ of 44 participating sites were not using any protocol for assessing analgesia and sedation, respectively. ${ }^{26}$

A link between systematic pain assessment and outcome in critical illness has been shown. ${ }^{27}$ Similarly, in another study including 34 ICUs in France and Belgium, it was seen that $14 \%$ patients reported current pain even after 3-16 months' post-ICU stay with greater traumatic stress. ${ }^{28}$ In our study, majority of patients had better outcome with lower pain scores. However, the patients were not followed to study the post-ICU pain recall.

To best of our knowledge, this is the only study that has evaluated the clinical utility of BPAT in assessing BP and PP and its management in a tertiary Indian ICU setting.

A limitation of the study was that pain assessment using BPAT could not be attributed to the outcome and length of the stay as different patients included in the study had different etiology. Moreover, we did not power our study to detect survival benefit or mortality. We did not correlate the BPAT score with the sedation level of the patients and did not use any other pain scale. However, GCS was noted in all the patients and patients with GCS $\leq 3$ who could be unresponsive to pain were excluded from the study. Moreover, it has been demonstrated that grimace, wince, moaning, verbal complaints, and clenched fists have no interaction between time and sedation level. ${ }^{4}$

Owing to opioid crisis and opioid withdrawal syndrome, further research is needed to study the impact of implementation of BPAT to guide pain assessment and management in either adjusting the opioid daily dosages or efficacy of an opioid-sparing ICU protocol. ${ }^{29,30}$

\section{Conclusion}

To conclude, our study suggests high clinical applicability of BPAT in assessing both BP and PP and its management in a tertiary ICU setting.

\section{Clinical Significance}

We recommend implementing BPAT in clinical practice for better pain control in ICU patients.

\section{References}

1. Puntillo K, White C, Morris A, Perdue S, Stanik-Hutt J, Thompson C, et al. Patients' perceptions and responses to procedural pain: results from thunder project II. Am J Crit Care 2001;10(4):238-251. DOI: 10.4037/ajcc2001.10.4.238.

2. Narayanan M, Venkataraju A, Jennings J. Analgesia in intensive care: part 1. BJA Education 2016;16(2):72-78. DOI: 10.1093/bjaceaccp/ mkv018.

3. Puntillo KA, Max A, Timsit JF, Vignoud L, Chanques G, Robleda G, et al. Determinants of procedural pain intensity in the intensive care unit. Am J Respir Crit Care Med 2014;189(1):39-47. DOI: 10.1164/ rccm.201306-11740C.

4. Gelinas C, Puntillo KA, Levin P, Azoulay E. The behavior pain assessment tool for critically ill adults: a validation study in 28 countries. Pain 2017;158(5):811-821. DOI: 10.1097/j.pain.0000000000000834.

5. Gelinas $C$. Nurses' evaluations of the feasibility and clinical utility of the critical care pain observational tool. Pain Manag Nurs 2010;11(2):115-125. DOI: 10.1016/j.pmn.2009.05.002.

6. Smart A. A multi-dimensional model of clinical utility. Int J Qual Health C 2006;18(5):377-382. DOI: 10.1093/intqhc/mzl034.

7. Rostad HM, Utne I, Grov EK, Puts M, Halvorsrud L. Measurement properties, feasibility and clinical utility of the Doloplus-2 pain scale in older adults with cognitive impairment: asystematic review. BMC Geriatr 2017;17(1):257-285. DOI: 10.1186/s12877-017-0643-9.

8. Richard LM, Williams V, Bernard F, Tsoller D, Gélinas C. Nurses' evaluations of the feasibility and clinical utility of the use of the critical-care pain observation tool-neuro in critically ill brain-injured patients. SNAHP 2019. 2. DOI: 10.31770/2561-7516.1045. 
9. Pathak A, Sharma S, Jensen MP. The utility and validity of pain intensity rating scales for use in developing countries. Pain Rep 2018(5):e672. DOI: 10.1097/PR9.0000000000000672.

10. Teasdale G, Allen D, Brennan P, McElhinney E, Mackinnon L. The Glasgow coma scale: an update after 40 years. Nurs Times 2014;110:12-16.

11. Breivik H, Borchgrevink PC, Allen SM, Rosseland LA, Romundstad $L$, Hals BEK. Assessment of pain. BJA 2008;101(1):17-24. DOI: 10.1093/ bja/aen103.

12. Gouda MA. Common pitfalls in reporting the use of SPSS software. Med Princ Pract 2015;24(3):300. DOI: 10.1159/000381953.

13. Santos IFA, DeSantana JM. Pain measurement techniques: spotlight on mechanically ventilated patients. J Pain Res 2018;11:2969-2980. DOI: $10.2147 / J P R . S 151169$.

14. Khanna P, Pandey RK, Chandralekha C, Sharma A, Pangasa N. Comparison between critical-care pain observation tool and physiologic indicators for pain assessment in the critically ill, mechanically ventilated adult patients. Saudi J Anaesth 2018;12(3):384-388. DOI: 10.4103/sja.SJA_642_17.

15. Barr J, Fraser GL, Puntillo K, Ely EW, Gélinas C, Dasta JF, et al. Clinical practice guidelines for the management of pain, agitation, and delirium in adult patients in the intensive care unit. Crit Care Med 2013;41(1):263-306. DOI: 10.1097/CCM.0b013e3182783b72.

16. Gélinas $C$, Arbour C. Behavioral and physiological indicators during a nociceptive procedure in conscious and unconscious mechanically ventilated adults: similar or different? J Crit Care 2009;24(4):628.e7-17. DOI: 10.1016/j.jcrc.2009.01.013.

17. Naithani U, Bajaj P, Chhabra S. Assessment of sedation and analgesia in mechanically ventilated patients in intensive care unit. Indian J Anaesth 2008;52:519.

18. Deborah BM, Karen SK, Mary EH-W, Florence I. Pain assessment in Non-communicative adult palliative care patients. NursClin North Am 2016;51(3):397-431. DOI: 10.1016/j.cnur.2016.05.009.

19. Gélinas C, Joffe AM, Szumita PM, Payen JF, Bérubé M, Shahiri T. S, et al. A psychometric analysis update of behavioral pain assessment tools for noncommunicative, critically ill adults. AACN Adv Crit Care 2019;30(4):365-387. DOI: 10.4037/aacnacc2019952.

20. Chanques G, Pohlman A, Molinari N, Jong AD, Jaber S, Kress JP. Psychometric comparison of three behavioural scales for the assessment of pain in critically ill patients unable to self-report. Crit Care 2014(5):18. DOI: 10.1186/cc14000.

21. Aïssaoui Y, Zeggwagh AA, Zekraoui A, Abidi K, Abouqal R. Validation of a behavioral pain scale in critically ill, sedated, and mechanically ventilated patients. Anesth Analg 2005;101(5):1470-1476. DOI: 10.1213/01.ANE.0000182331.68722.FF.

22. Czarnecki ML, Turner HN, Collins PM, Doellman D, Wrona S, Reynolds J. Procedural pain management: a position statement with clinical practice recommendations. Pain Manag Nurs 2011;12(2):95-111. DOI: 10.1016/j.pmn.2011.02.003.

23. Payen J-F, Bru O, Bosson J-L, Lagrasta A, Novel E, Deschaux I, et al. Assessing pain in critically ill sedated patients by using a behavioral pain scale. Crit Care Med 2001;29(12):2258-2263. DOI: 10.1097/00003246-200112000-00004.

24. Gélinas C, Fillion L, Puntillo KA, Viens C, Fortier M. Validation of the critical-care pain observation tool in adult patients. Am J Crit Care 2006;15(4):420-427. DOI: 10.4037/ajcc2006.15.4.420.

25. Young J, Siffleet J, Nikoleeti S, Shaw T. Use of behavioural pain scale to assess pain in ventilated, unconscious and/or sedated patients. IntensCrit care nur 2006;22(1):32-39. DOI: 10.1016/j.iccn.2005.04.004.

26. Payen JF, Chanques G, Mantz J, Hercule C, Auriant I, Leguillou JL, et al. Current practices in sedation and analgesia for mechanically ventilated critically ill patients: a prospective multicenter patientbased study. Anesth 2007;106(4):687-695. DOI: 10.1097/01. anes.0000264747.09017.da.

27. Georgiou E, Hadjibalassi M, Lambrinou E, Andreou P, Papathanassoglou E. The impact of pain assessment on critically ill patients' outcomes: a systematic review. Biomed Res Int 2015. DOI: http://dx.doi. org/10.1155/2015/503830.

28. Puntillo AK, Max A, Chaize M, Chanques G, Azoulay E. Patient recollection of ICU procedural pain and post ICU burden: the memory study. Crit Care Med 2016;44(11):1988-1995. DOI: 10.1097/ CCM.0000000000001875.

29. Gelinas C. Pain management challenges in acute and critically ill patients. AACN Adv Crit Care 2019;30(4):318-319. DOI: 10.4037/ aacnacc2019132.

30. Arroyo-Novoa CM, Figueroa-Ramos MI, Puntillo KA. Opioid and benzodiazepine iatrogenic withdrawal syndrome in patients in the intensive care unit. AACN Adv Crit Care 2019;30(4):353-364. DOI 10.4037/aacnacc2019267. 